\title{
Some improvements on the Ky Fan theorem for tensors
}

\author{
Mohsen Tourang ${ }^{1} \cdot$ Mostafa Zangiabadi $^{1}$ \\ Received: 3 August 2020 / Revised: 18 November 2020 / Accepted: 3 December 2020 / \\ Published online: 1 March 2021 \\ (c) Springer Nature Switzerland AG part of Springer Nature 2021
}

\begin{abstract}
The improvements of Ky Fan theorem are given for tensors. First, based on Brauer-type eigenvalue inclusion sets, we obtain some new Ky Fan-type theorems for tensors. Second, by characterizing the ratio of the smallest and largest values of a Perron vector, we improve the existing results. Third, some new eigenvalue localization sets for tensors are given and proved to be tighter than those presented by $\mathrm{Li}$ and $\mathrm{Ng}$ (Numer Math 130(2):315-335, 2015) and Wang et al. (Linear Multilinear Algebra 68(9):1817-1834, 2020). Finally, numerical examples are given to validate the efficiency of our new bounds.
\end{abstract}

Keywords Ky Fan theorem - Tensor eigenvalue · Eigenvalue inclusion set

Mathematics Subject Classification $15 \mathrm{~A} 18 \cdot 15 \mathrm{~A} 69 \cdot 15 \mathrm{~A} 42$

\section{Introduction}

Let $\mathbb{C}(\mathbb{R})$ be the set of all complex (real) numbers, and $\mathbb{C}^{n}\left(\mathbb{R}^{n}\right)$ be the set of all dimension $n$ complex (real) vectors. An order $m$ dimension $n$ complex (real) tensor $\mathcal{A}=\left(a_{i_{1} i_{2} \ldots i_{m}}\right)$, denoted by $\mathcal{A} \in \mathbb{C}^{[m, n]}\left(\mathcal{A} \in \mathbb{R}^{[m, n]}\right.$, respectively), consists of $n^{m}$ entries:

$$
a_{i_{1} i_{2} \ldots i_{m}} \in \mathbb{C}(\mathbb{R}), \quad \forall i_{j}=1, \ldots, n, \quad j=1, \ldots, m .
$$

A tensor $\mathcal{A}=\left(a_{i_{1} i_{2} \ldots i_{m}}\right) \in \mathbb{R}^{[m, n]}$ is called nonnegative (positive) if:

$$
a_{i_{1} i_{2} \ldots i_{m}} \geq 0\left(a_{i_{1} i_{2} \ldots i_{m}}>0\right), \quad \forall i_{j}=1, \ldots, n, \quad j=1, \ldots, m .
$$

Tensors have many similarities with matrices and many related results of matrices such as eigenvector and eigenvalue can be extended to higher order tensors (e.g., see Qi and Luo 2017; Wei and Ding 2017).

Communicated by Jinyun Yuan.

Mostafa Zangiabadi

zangiabadi1@gmail.com

Mohsen Tourang

mohsentourang@gmail.com

1 Department of Mathematics, University of Hormozgan, P.O. Box 3995, Bandar Abbas, Iran 
For a vector $x \in \mathbb{C}^{n}$, we use $x_{i}$ to denote its components and $x^{[m-1]}$ to denote a vector in $\mathbb{C}^{n}$, such that:

$$
x_{i}^{[m-1]}=x_{i}^{m-1} \text { for all } i .
$$

$\mathcal{A} x^{m-1}$ denotes a vector in $\mathbb{C}^{n}$, whose $i$ th component is:

$$
\left(\mathcal{A} x^{m-1}\right)_{i}=\sum_{i_{2}, i_{3}, \ldots ., i_{m}=1}^{n} a_{i i_{2} \ldots i_{m}} x_{i_{2}} \ldots x_{i_{m}} .
$$

A pair $(\lambda, x) \in \mathbb{C} \times\left(\mathbb{C}^{n} \backslash\{0\}\right)$ is called an eigenpair (eigenvalue-eigenvector pair) of $\mathcal{A}$ if they satisfy:

$$
\mathcal{A} x^{m-1}=\lambda x^{[m-1]} .
$$

Specifically, $(\lambda, x)$ is called an H-eigenpair if $(\lambda, x) \in \mathbb{R} \times \mathbb{R}^{n} \backslash\{0\}$.

With the introduction of tensor eigenvalue, it is of interest to investigate eigenvalue inclusion regions, i.e., to find the regions including all eigenvalues in the complex plane. The first work owes to Liqun Qi. Qi (2005) gave an eigenvalue inclusion set for real symmetric tensors, which is a generalization of the well-known Gershgorin set of matrices (Horn and Johnson 2012). Subsequently, due to its fundamental applications in various fields, many researchers are interested in investigating eigenvalue inclusion regions for tensors, e.g., Bu et al. (2017), Li and Li (2016a), Li et al. (2014, 2020), Xu et al. (2019), etc.

In 1958, Fan (1958) obtained the famous Ky Fan theorem that plays an important role in the study of the nonnegative eigenvalue problem. Li and Huang (2005) presented an improvement on the Ky Fan theorem for any weakly irreducible $n \times n$ complex matrix $A$. Yang and Yang (2010) generalized the Ky Fan-type theorem from matrices to tensors. Later, Li and Ng (2015) improved it, and recently, Wang et al. (2020) proved some new Ky Fan-type theorems for weakly irreducible nonnegative tensors with Brualdi-type eigenvalue inclusion set. In this paper, we provide some new improvements on the Ky Fan theorem, which improves the existing bounds.

\section{Preliminaries}

We begin this section with some definitions and statements that are needed for the main results of our work which are taken from $\mathrm{Bu}$ et al. (2017), $\mathrm{Li}$ and $\mathrm{Li}$ (2016a,b), Li et al. (2014), Qi and Luo (2017), and Yang and Yang (2010).

Definition 1 Let $\mathcal{A}$ be a tensor of order $m$ dimension $n$.

(i) We call $\sigma(\mathcal{A})$ as the set of all eigenvalues of $\mathcal{A}$ and the spectral radius of $\mathcal{A}$ is denoted by:

$$
\rho(\mathcal{A})=\max \{|\lambda|: \lambda \in \sigma(A)\} .
$$

(ii) We call a tensor $\mathcal{A}$ reducible if there exists a nonempty proper index subset $I \subset\langle n\rangle:=$ $\{1,2, \ldots, n\}$, such that:

$$
a_{i_{1} i_{2} \ldots i_{m}}=0, \quad \forall i_{1} \in I, \quad i_{2}, \ldots, i_{m} \notin I .
$$

If $\mathcal{A}$ is not reducible, then we call $\mathcal{A}$ irreducible. 
(iii) We call a tensor $\mathcal{A}$ nonnegative weakly irreducible, if, for any nonempty proper index subset $I \subset\langle n\rangle$, there is at least an entry $a_{i_{1} i_{2} \ldots i_{m}}>0$, where $i_{1} \in I$, and at least an $i_{j} \notin I, \quad j=2, \ldots, m$.

(iv) We denote by $\delta_{i_{1} i_{2} \ldots i_{m}}$, the Kronecker symbol for the case of $m$ indices, that is:

$$
\delta_{i_{1} i_{2} \ldots i_{m}}= \begin{cases}1 & i_{1}=i_{2}=\cdots=i_{m} \\ 0 & \text { otherwise. }\end{cases}
$$

Let us recall the Perron-Frobenius theorem for irreducible nonnegative tensors given in Yang and Yang (2010).

Theorem 1 (see Theorem 2.2 of Yang and Yang 2010) Suppose that $\mathcal{A}$ is an irreducible nonnegative tensor of order $m$ dimension $n$. Then, $\rho(\mathcal{A})>0$ is an eigenvalue of $\mathcal{A}$ with a positive eigenvector $x$ corresponding to it.

Remark 1 It is noted that the spectral radius $\rho(\mathcal{A})$ is the largest H-eigenvalue for the nonnegative tensor Yang and Yang (2010).

Note that $\rho(\mathcal{A})$ and $x$ in Theorem 1 are called the Perron root and the Perron vector of $\mathcal{A}$, respectively, and $(\rho(\mathcal{A}), x)$ is regarded as a Perron eigenpair.

Definition 2 If $\mathcal{A}=\left(a_{i_{1} i_{2} \ldots i_{m}}\right)$ is a real tensor of order $m$ dimension $n$ and $D$ is a matrix, then the product of $\mathcal{A}$ and $D$ is defined as follows:

$$
\mathcal{A}_{D}=\mathcal{A} . D^{-(m-1)} \cdot \overbrace{D \ldots D}^{m-1}
$$

where

$$
\left(\mathcal{A}_{D}\right)_{i_{1} i_{2} \ldots i_{m}}=\sum_{j_{1}, j_{2}, \ldots, j_{m}=1}^{n} a_{j_{1} \ldots j_{m}} D_{i_{1}, j_{1}}^{-(m-1)} D_{i_{2}, j_{2}} \ldots D_{i_{m}, j_{m}} .
$$

If $D$ is a diagonal matrix, then:

$$
\left(\mathcal{A}_{D}\right)_{i_{1} i_{2} \ldots i_{m}}=a_{i_{1} \ldots i_{m}} D_{i_{1}, i_{1}}^{-(m-1)} D_{i_{2}, i_{2} \ldots D_{i_{m}, i_{m}}}
$$

Remark 2 (Yang and Yang 2010) In fact, if:

$$
\mathcal{A}_{D}=\mathcal{A} \cdot D^{-(m-1)} \cdot \overbrace{D \ldots D}^{m-1}
$$

where $D$ is a diagonal nonsingular matrix, and then, $\mathcal{A}_{D}$ and $\mathcal{A}$ have the same eigenvalues. If $\lambda$ is an eigenvalue of $\mathcal{A}_{D}$ with corresponding eigenvector $x$, then $\lambda$ is also an eigenvalue of $\mathcal{A}$ with corresponding eigenvector $D x$; if $\mu$ is an eigenvalue of $\mathcal{A}$ with corresponding eigenvector $y$, then $\mu$ is an eigenvalue of $\mathcal{A}_{D}$ with corresponding eigenvector $D^{-1} y$.

In recent years, the spectral theory of tensors has attracted much attention. In 2005, Qi and Luo (2017) gave an eigenvalue localization set for real symmetric tensors, which is a generalization of the well-known Gershgorin's eigenvalue localization theorem of matrices (Horn and Johnson 2012; Varga 2004). This result has also been generalized to general tensors (Li et al. 2014; Yang and Yang 2010). 
Theorem 2 (Li et al. 2014, Theorem 1.1) Let $\mathcal{A}=\left(a_{i_{1} i_{2} \ldots i_{m}}\right)$ be a tensor of order $m$ dimension n. Then:

$$
\sigma(\mathcal{A}) \subseteq \Gamma(\mathcal{A})=\bigcup_{i \in\langle n\rangle} \Gamma_{i}(\mathcal{A})
$$

where $\Gamma_{i}(\mathcal{A})=\left\{z \in \mathbb{C}:\left|z-a_{i i \ldots i}\right| \leq r_{i}(\mathcal{A})\right\}$, and $r_{i}(\mathcal{A})=\sum_{\substack{i_{2}, \ldots i_{m}=1 \\ \delta_{i i_{2} \ldots i_{m}=0}}}^{n}\left|a_{i i_{2} \ldots i_{m}}\right|$.

The well-known Brauer's eigenvalue inclusion set of matrices was given in Brauer (1947), which is always contained in the Gershgorin set. Recently, Li et al. (2014) extended the Brauer's eigenvalue inclusion set of matrices to tensors, and gave the following Brauer-type eigenvalue inclusion set for tensors.

Theorem 3 (Li et al. 2014, Theorem 2.1) Let $\mathcal{A}=\left(a_{i_{1} i_{2} \ldots i_{m}}\right)$ be a tensor of order $m$ dimension $n$, and then:

$$
\sigma(\mathcal{A}) \subseteq \mathcal{F}(\mathcal{A})=\bigcup_{i, j \in\langle n\rangle, j \neq i} \mathcal{F}_{i, j}(\mathcal{A})
$$

where

$$
\mathcal{F}_{i, j}=\left\{z \in \mathbb{C}:\left(\left|z-a_{i i \ldots i}\right|-r_{i}^{j}(\mathcal{A})\right)\left|z-a_{j j \ldots j}\right| \leq\left|a_{i j \ldots j}\right| r_{j}(\mathcal{A})\right\},
$$

and

$$
r_{i}^{j}(\mathcal{A}):=\sum_{\substack{i_{2}, \ldots i_{m}=1 \\ \delta_{i i_{2} \ldots i_{m}=0} \\ \delta_{j i_{2} \ldots i_{m}=0}}}^{n}\left|a_{i i_{2} \ldots i_{m}}\right|=r_{i}(\mathcal{A})-\left|a_{i j \ldots j}\right|
$$

Very recently, Bu et al. (2017) gave another Brauer-type eigenvalue localization sets of tensors as follows, which is proved to be tighter than the sets $\Gamma(\mathcal{A})$ and $\mathcal{F}(\mathcal{A})$.

Theorem 4 (Bu et al. 2017, Theorem 3.1) Let $\mathcal{A}=\left(a_{i_{1} i_{2} \ldots i_{m}}\right)$ be a tensor of order $m$ dimension $n$. Then, every eigenvalue of $\mathcal{A}$ lies in the region:

$$
\mathfrak{B}(\mathcal{A})=\bigcup_{\substack{i, j \in\langle n\rangle \\ i \neq j}}\left\{z \in \mathbb{C}:\left|z-a_{i i \ldots i}\right|^{m-1}\left|z-a_{j j \ldots j}\right| \leq r_{i}(\mathcal{A})^{m-1} r_{j}(\mathcal{A})\right\} .
$$

Theorem 5 (Bu et al. 2017, Theorem 3.3) Let $\mathcal{A}=\left(a_{i_{1} i_{2} \ldots i_{m}}\right)$ be a tensor of order $m$ dimension $n$, and $r_{i}(\mathcal{A}) \neq 0, \quad i=1, \ldots, n$. Then, every eigenvalue of $\mathcal{A}$ lies in the region:

$$
\mathcal{Z}(\mathcal{A})=\bigcup_{\substack{a_{i_{1} i_{2} \ldots i_{m}} \neq 0 \\ \delta_{i_{1} i_{2} \ldots i_{m}}=0}}^{\bigcup}\left\{z \in \mathbb{C}: \prod_{j=1}^{m}\left|z-a_{i_{j} i_{j} \ldots i_{j}}\right| \leq \prod_{j=1}^{m} r_{i_{j}}(\mathcal{A})\right\} .
$$

To find such relationships between other Brauer-type eigenvalue localization sets, see (Li and Li 2016b; Xu et al. 2019). 


\section{Improvements of Ky Fan theorem}

The Ky Fan theorem (e.g., see Theorem 8.2.12 of Horn and Johnson 2012) plays an important role in estimating the eigenvalues of matrices. Yang and Yang (2010, Theorem 4.1), gave the Ky Fan-type inequality for tensors as follows:

Theorem 6 Let $\mathcal{A}=\left(a_{i_{1} i_{2} \ldots i_{m}}\right)$ and $\mathcal{B}=\left(b_{i_{1} i_{2} \ldots i_{m}}\right)$ be complex tensors of order $m$ dimension $n$ with $|\mathcal{A}| \leq \mathcal{B}$. If

$$
\mathcal{K}^{(i)}(\mathcal{A})=\left\{z \in \mathbb{C}:\left|z-a_{i i \ldots i}\right| \leq \rho(\mathcal{B})-b_{i i \ldots i}\right\}, \quad i=1, \ldots, n,
$$

denotes the $i$ th Ky Fan disk for $\mathcal{A}$, then, the union of these $n$ Ky Fan disks contains all eigenvalues of $\mathcal{A}$, that is:

$$
\sigma(\mathcal{A}) \subseteq \mathcal{K}(\mathcal{A}):=\bigcup_{i=1}^{n} \mathcal{K}^{(i)}(\mathcal{A})
$$

The Ky Fan-type theorem naturally extends from matrices to tensors (see Yang and Yang 2010). Later, $\mathrm{Li}$ and $\mathrm{Ng}$ (2015) improved it by applying the ratio of the entries in the Perron vector, and recently, Wang et al. (2020) proved some new Ky Fan-type theorems for weakly irreducible nonnegative tensors with Brualdi-type eigenvalue inclusion sets. In the following sections, we will present some improvements of Ky Fan theorem. Also, we will give examples showing that our results are sharp.

\subsection{Improvements of Ky Fan theorem based on Brualdi-type sets}

In this section, an improvement of Ky Fan theorem is suggested by Brauer-type eigenvalue inclusion set $\mathcal{Z}(\mathcal{A})$, as defined in Theorem 5 .

Theorem 7 Let $\mathcal{A}=\left(a_{i_{1} i_{2} \ldots i_{m}}\right)$ and $\mathcal{B}=\left(b_{i_{1} i_{2} \ldots i_{m}}\right)$ be complex tensors of order $m$ dimension $n$. If $|\mathcal{A}| \leq \mathcal{B}$, and $r_{i}(\mathcal{A}) \neq 0, \quad i=1, \ldots, n$, then:

$$
\sigma(\mathcal{A}) \subseteq \bigcup_{\substack{a_{i_{1} i_{2} \ldots i_{m}} \neq 0 \\ \delta_{i_{1} i_{2} \ldots i_{m}=0}=0}}\left\{z \in \mathbb{C}: \prod_{j=1}^{m}\left|z-a_{i_{j} i_{j} \ldots i_{j}}\right| \leq \prod_{j=1}^{m}\left(\rho(\mathcal{B})-b_{i_{j} i_{j} \ldots i_{j}}\right)\right\}:=\mathcal{Z}_{1}(\mathcal{A}) .
$$

Proof We may assume that $\mathcal{B}>0$ (if some entries of $\mathcal{B}$ are zero, we may consider $\mathcal{B}_{\varepsilon} \equiv$ $\left(b_{i_{1} i_{2} \ldots i_{m}}+\varepsilon\right)$, for $\varepsilon>0 ; \mathcal{B}_{\varepsilon}>|\mathcal{A}|$ and $\rho\left(\mathcal{B}_{\varepsilon}\right)-\left(b_{i i \ldots i}+\varepsilon\right) \rightarrow \rho(\mathcal{B})-b_{i i \ldots i}$ when $\varepsilon \rightarrow 0$ ). By Perron-Frobenius theorem (Chang et al. 2008), there exists a positive vector $x=\left(x_{1}, x_{2}, \ldots, x_{n}\right)^{T}$, such that:

$$
\mathcal{B} x^{m-1}=\rho(\mathcal{B}) x^{[m-1]} .
$$

Let $X:=\operatorname{diag}\left(x_{1}, x_{2}, \ldots, x_{n}\right)$, and $\mathcal{A}_{X}:=\mathcal{A} X^{-(m-1)} \overbrace{X \ldots X}^{m-1}$. Then, by Remark $2 \sigma(\mathcal{A})=$ $\sigma\left(\mathcal{A}_{X}\right)$ and $\left|\left(\mathcal{A}_{X}\right)_{i i \ldots i}\right|=\left|a_{i i \ldots i}\right|<b_{i i \ldots i}$, for all $i \in\langle n\rangle$.

By Theorem 5 for any eigenvalue $\lambda$ of $\mathcal{A}_{X}$, we have:

$$
\prod_{j=1}^{m}\left|\lambda-a_{i_{j} i_{j} \ldots i_{j}}\right| \leq \prod_{j=1}^{m} r_{i_{j}}\left(\mathcal{A}_{X}\right)=\prod_{j=1}^{m}\left(\sum_{\substack{i_{2}, \ldots i_{m}=1 \\ \delta_{i_{j} i_{2} \ldots i_{m}=0}}}^{n} \frac{\left|a_{i_{j} i_{2} \ldots i_{m}}\right| x_{i_{2}} \ldots x_{i_{m}}}{x_{i_{j}}^{m-1}}\right)
$$




$$
\begin{aligned}
& \leq \prod_{j=1}^{m}\left(\sum_{\substack{i_{2}, \ldots i_{m}=1 \\
\delta_{i_{j} i_{2} \ldots i_{m}=0}}}^{n} \frac{b_{i_{j} i_{2} \ldots i_{m}} x_{i_{2}} \ldots x_{i_{m}}}{x_{i_{j}}^{m-1}}\right) \\
& =\prod_{j=1}^{m}\left(\sum_{\substack{i_{2}, \ldots i_{m}=1 \\
\delta_{i_{j} i_{2} \ldots i_{m}=0}}}^{n} \frac{b_{i_{j} i_{2} \ldots i_{m}} x_{i_{2}} \ldots x_{i_{m}}}{x_{i_{j}}^{m-1}}+b_{i_{j} i_{j} \ldots i_{j}}-b_{i_{j} i_{j} \ldots i_{j}}\right) \\
& =\prod_{j=1}^{m}\left(\sum_{i_{2}, \ldots i_{m}=1}^{n} \frac{b_{i_{j} i_{2} \ldots i_{m}} x_{i_{2}} \ldots x_{i_{m}}}{x_{i_{j}}^{m-1}}-b_{i_{j} i_{j} \ldots i_{j}}\right) \\
& =\prod_{j=1}^{m}\left(\rho(\mathcal{B})-b_{i_{j} i_{j} \ldots i_{j}}\right) \text {. }
\end{aligned}
$$

Thus, we have:

$$
\sigma(\mathcal{A}) \subseteq \bigcup_{\substack{a_{i_{1} i_{2} \ldots i_{m}} \neq 0 \\ \delta_{i_{1} i_{2} \ldots i_{m}}=0}}\left\{z \in \mathbb{C}: \prod_{j=1}^{m}\left|z-a_{i_{j} i_{j} \ldots i_{j}}\right| \leq \prod_{j=1}^{m}\left(\rho(\mathcal{B})-b_{i_{j} i_{j} \ldots i_{j}}\right)\right\}:=\mathcal{Z}_{1}(\mathcal{A})
$$

The theorem is proved.

Remark 3 Note that in Theorem 7, $\sigma(\mathcal{A}) \subseteq \mathcal{Z}_{1}(\mathcal{A})$ is also obtained by removing the condition $r_{i}(\mathcal{A}) \neq 0, \quad i=1, \ldots, n$.

Similarly, using Theorem 4 and the method of proof employed in Theorem 7, we have the following:

Corollary 1 Let $\mathcal{A}=\left(a_{i_{1} i_{2} \ldots i_{m}}\right)$ and $\mathcal{B}=\left(b_{i_{1} i_{2} \ldots i_{m}}\right)$ be complex tensors of order $m$ dimension $n$ with $|\mathcal{A}| \leq \mathcal{B}$. Then, every eigenvalue of $\mathcal{A}$ lies in the region:

$$
\begin{aligned}
& \bigcup_{\substack{i, j=1 \\
i \neq j}}^{n}\left\{z \in C:\left|z-a_{i i \ldots i}\right|^{m-1} \cdot\left|z-a_{j j \ldots j}\right| \leq\left(\rho(\mathcal{B})-b_{i i \ldots i}\right)^{m-1}\left(\rho(\mathcal{B})-b_{j j \ldots j}\right)\right\} \\
& :=\mathfrak{B}_{1}(\mathcal{A}) .
\end{aligned}
$$

\subsection{Improvements of Ky Fan theorem based on the ratio of the entries in the Perron vector}

$\mathrm{Li}$ and $\mathrm{Ng}(2015)$ considered lower bound the problem of estimating the ratio $\frac{x_{\min }}{x_{\max }}$ for an irreducible nonnegative tensor $\mathcal{A}$ as follows:

$$
\kappa(\mathcal{A}) \leq \frac{x_{\min }}{x_{\max }}
$$


where $x_{\min }=\min _{1 \leq i \leq n} x_{i}$ and $x_{\max }=\max _{1 \leq i \leq n} x_{i}$, and:

$$
\kappa(\mathcal{A})=\max _{2 \leq k, k^{\prime} \leq m} \min _{\substack{1 \leq i_{1}, i_{1}^{\prime} \leq n \\ 1 \leq i_{k}=i_{k}^{\prime} \leq n}} \frac{\underbrace{i_{2}, \ldots, i_{m}}_{\text {except } i_{k}}=1}{\sum_{\text {except } i_{k}^{\prime}}^{i_{2}^{\prime}, \ldots, i_{m}^{\prime}}=1} a_{i_{1}^{\prime}, \ldots, i_{m}}^{\sum_{i_{1}^{\prime}, \ldots, i_{m}^{\prime}}} .
$$

Using similar technique, the lower bound of (4) can be further improved as below (see Lemma 4.1 in Wang et al. 2020):

$$
\iota(\mathcal{A}) \leq\left(\frac{x_{\min }}{x_{\max }}\right)^{2}
$$

where

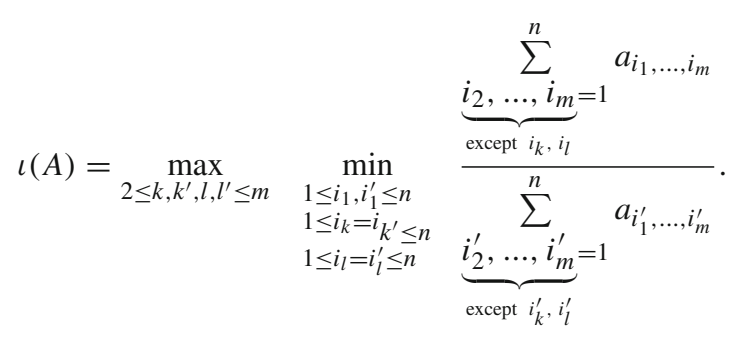

Also, using similar technique as presented in Wang et al. (2020, Lemma 4.1) (or Li and $\mathrm{Ng}$ 2015, Lemma 3.2), we obtain the lower bound for the ratio of the smallest and largest entries in a Perron vector.

Lemma 1 Let $\mathcal{A}$ be a weakly irreducible nonnegative tensor of order $m$ dimension $n$ with maximal eigenvector $x$. Then:

$$
\tau(\mathcal{A})=\max _{1 \leq k \leq m-1} \tau^{(k)}(\mathcal{A}) \leq \frac{x_{\min }}{x_{\max }},
$$

where

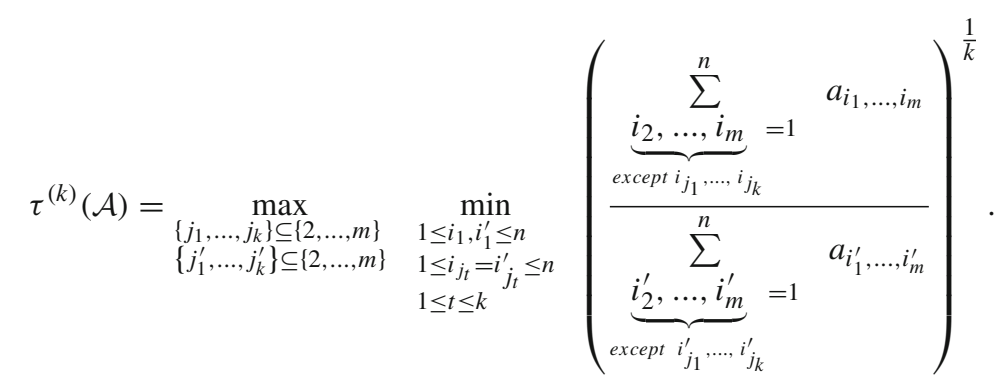

Proof Let $x_{\alpha}=\min x_{i}$ and $x_{\beta}=\max x_{i}$, Then, by (3), we have:

$$
\rho(\mathcal{A})\left(x_{\alpha}\right)^{m-1}=\sum_{i_{2}, \ldots, i_{m}=1}^{n} a_{\alpha, i_{2}, \ldots, i_{m}} x_{i_{2}} \ldots x_{i_{m}}
$$




$$
\geq\left(\sum_{i_{2}, \ldots, i_{m}=1}^{n} a_{\alpha, i_{2}, \ldots, i_{m}} x_{i_{j_{1}}} \ldots x_{i_{j_{k}}}\right) x_{\alpha}^{m-1-k},
$$

where $\left\{j_{1}, \ldots, j_{k}\right\} \subseteq\{2, \ldots, m\}$. It implies that:

$$
\rho(\mathcal{A})\left(x_{\alpha}\right)^{k} \geq \sum_{i_{2}, \ldots, i_{m}=1}^{n} a_{\alpha, i_{2}, \ldots, i_{m}} x_{i_{j_{1}} \ldots x_{i_{j_{k}}}} .
$$

Similarly, we get:

$$
\rho(\mathcal{A})\left(x_{\beta}\right)^{k} \leq \sum_{i_{2}, \ldots, i_{m}=1}^{n} a_{\beta, i_{2}, \ldots, i_{m}} x_{i_{j_{1}} \ldots x_{i_{j_{k}}}} .
$$

Therefore:

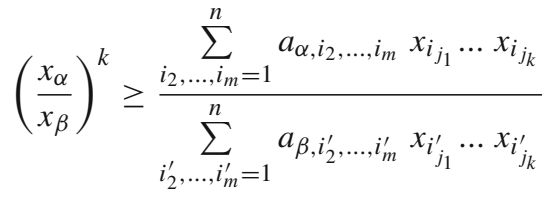

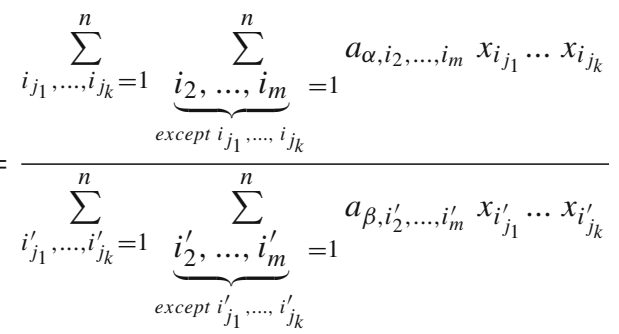

$$
\begin{aligned}
& \geq \min _{\substack{1 \leq i_{j_{t}} i_{j_{t}}^{\prime} \leq n \\
1 \leq t \leq k}} \frac{\underbrace{i_{2}, \ldots, i_{m}}_{\text {except } i_{j_{1}}, \ldots, i_{j_{k}}}=1}{\sum_{\text {except }{ }^{\prime}{ }_{j_{1}}, \ldots, i_{j_{k}}^{\prime}}^{i_{2}^{\prime}, \ldots, i_{m}^{\prime}}=1} a_{\beta, i_{2}, \ldots, i_{m}} a_{\beta, i_{2}, \ldots, i_{m}^{\prime}} \\
& \geq \min _{1 \leq i_{1}=i_{1}^{\prime} \leq n} \min _{\substack{1 \leq i_{j_{t}}=i_{j_{t}}^{\prime} \leq n \\
1 \leq t \leq k}} \frac{\underbrace{\sum_{\text {except } i_{j_{1}}^{\prime}, \ldots, i_{j_{k}}^{\prime}, \ldots, i_{m}}^{n}}_{\text {except } i_{j_{1}, \ldots, i_{j_{k}}}}=1}{\sum_{i_{i_{1}, i_{2}, \ldots, i_{m}}}^{i_{2}^{\prime}, \ldots, i_{m}^{\prime}}=1} a_{i_{1}^{\prime}, i_{2}^{\prime}, \ldots, i_{m}^{\prime}}
\end{aligned}
$$

which proves the lemma.

Based on (4), Li and $\mathrm{Ng}$ (2015, Theorem 3.3) proposed the following theorem. 
Theorem 8 Let $\mathcal{A}$ and $\mathcal{B}$ be nonnegative tensors of order $m$ dimension $n$. If $\mathcal{B}$ is irreducible with $|\mathcal{A}| \leq \mathcal{B}$. Then, for any eigenvalue $\lambda$ of $\mathcal{A}$, there exists $i$, such that:

$$
\left|\lambda-a_{i \ldots i}\right| \leq \rho(\mathcal{B})-b_{i i \ldots i}-(\kappa(\mathcal{B}))^{m-1} r_{i}(\mathcal{E}),
$$

where $\mathcal{E}=\mathcal{B}-|\mathcal{A}|$ and $\kappa(\mathcal{B})$ is given in (5).

Recently, Wang et al. (2020, Theorem 4.6) improved the eigenvalue inclusion set (10), using (6) and $v(\mathcal{A})=\frac{l}{\rho(\mathcal{A})-R_{\min }(\mathcal{A})+l}$, which $l=\min _{\delta\left(i_{1}, \ldots, i_{m}\right)=0} a_{i_{1} \ldots i_{m}}$ and $R_{i}(\mathcal{A})=$ $\sum_{i_{2}, \ldots, i_{m}=1}^{n}\left|a_{i, i_{2}, \ldots, i_{m}}\right|$.

Theorem 9 Let $\mathcal{A}$ be a weakly irreducible tensors of order $m$ dimensional $n$, and $\mathcal{B}$ be a tensors of order $m$ dimensional $n$ with $|\mathcal{A}| \leq \mathcal{B}$. Then, for any eigenvalue $\lambda$ of $\mathcal{A}$, there exists $\gamma \in C(\mathcal{A})$, such that:

$$
\prod_{i \in \gamma}\left|\lambda-a_{i \ldots i}\right| \leq \prod_{i \in \gamma}\left(\rho(\mathcal{B})-b_{i i \ldots i}\right)-P^{|\gamma|} \prod_{i \in \gamma} r_{i}(\mathcal{E})
$$

where $P=\max \left\{(\kappa(\mathcal{B}))^{m-1},(\iota(\mathcal{B}))^{(m-1) / 2}, v(\mathcal{B})\right\}, \quad \mathcal{E}=\mathcal{B}-|\mathcal{A}|$ and $C(\mathcal{A})$ is the set of circuits of digraph $\mathcal{A}$ and $|\gamma|$ denotes the length of circuit $\gamma$.

Based on Brauer-type eigenvalue inclusion sets (Theorem 5) and estimating the ratio of the smallest and largest values of a Perron vector (Lemma 1), we can obtain the following Ky Fan-type theorem.

Theorem 10 Let $\mathcal{A}=\left(a_{i_{1} i_{2} \ldots i_{m}}\right)$ and $\mathcal{B}=\left(b_{i_{1} i_{2} \ldots i_{m}}\right)$ be complex tensors of order $m$ dimension $n$ with $|\mathcal{A}| \leq \mathcal{B}$. Then, every eigenvalue of $\mathcal{A}$ lies in the region:

$$
\underset{\substack{a_{i_{1} i_{2} \ldots i_{m}} \neq 0 \\ \delta_{i_{1} i_{2} \ldots i_{m}}=0}}{\bigcup}\left\{z \in \mathbb{C}: \prod_{j=1}^{m}\left|z-a_{i_{j} i_{j} \ldots i_{j}}\right| \leq \prod_{j=1}^{m}\left(\rho(\mathcal{B})-b_{i_{j} i_{j} \ldots i_{j}}-(\tau(\mathcal{B}))^{m-1} r_{i_{j}}(\mathcal{E})\right)\right\}:=\mathcal{Z}_{2}(\mathcal{A}),
$$

where $\mathcal{E}=\mathcal{B}-|\mathcal{A}|$ and $\tau(\mathcal{B})$ is given in $(8)$.

Proof The proof of this theorem runs parallel to proof for the corresponding part of Theorem 7. We may assume that $\mathcal{B}>0$. By Perron-Frobenius theorem (Chang et al. 2008), there exists a positive vector $x=\left(x_{1}, x_{2}, \ldots, x_{n}\right)^{T}$, such that:

$$
\mathcal{B} x^{m-1}=\rho(\mathcal{B}) x^{[m-1]} .
$$

Let $X:=\operatorname{diag}\left(x_{1}, x_{2}, \ldots, x_{n}\right), \mathcal{A}_{X}:=\mathcal{A} X^{-(m-1)} \overbrace{X \ldots X}^{m-1}$, and $\mathcal{B}_{X}:=\mathcal{B} X^{-(m-1)} \overbrace{X \ldots X}^{m-1}$. Then, by Remark 2 for all $i \in N$, we have:

$$
\begin{gathered}
\sigma(\mathcal{A})=\sigma\left(\mathcal{A}_{X}\right), \quad\left(\mathcal{A}_{X}\right)_{i i \ldots i}=a_{i i \ldots i}, \\
\sigma(\mathcal{B})=\sigma\left(\mathcal{B}_{X}\right), \quad\left(\mathcal{B}_{X}\right)_{i i \ldots i}=b_{i i \ldots i} .
\end{gathered}
$$

Let $\mathcal{E}=\mathcal{B}-|\mathcal{A}|$ and $\mathcal{E}_{X}=\mathcal{B}_{X}-|\mathcal{A}|_{X}$. By Theorem 5 for any eigenvalue $\lambda$ of $\mathcal{A}_{X}$, we have:

$$
\begin{aligned}
\prod_{j=1}^{m}\left|\lambda-a_{i_{j} i_{j} \ldots i_{j}}\right| & \leq \prod_{j=1}^{m} r_{i_{j}}\left(\mathcal{A}_{X}\right)=\prod_{j=1}^{m} r_{i_{j}}\left(\mathcal{B}_{X}-\mathcal{E}_{X}\right) \\
& =\prod_{j=1}^{m}\left(r_{i_{j}}\left(\mathcal{B}_{X}\right)-r_{i_{j}}\left(\mathcal{E}_{X}\right)\right)
\end{aligned}
$$




$$
\begin{aligned}
& =\prod_{j=1}^{m}\left(\rho(\mathcal{B})-b_{i_{j} i_{j} \ldots i_{j}}-\sum_{\substack{i_{2}, \ldots i_{m}=1 \\
\delta_{i_{j} i_{2} \ldots i_{m}=0}}}^{n} \frac{\mathcal{E}_{i_{j} i_{2} \ldots i_{m}} x_{i_{2}} \ldots x_{i_{m}}}{x_{i_{j}}^{m-1}}\right) \\
& \leq \prod_{j=1}^{m}\left(\rho(\mathcal{B})-b_{i_{j} i_{j} \ldots i_{j}}-\sum_{\substack{i_{2}, \ldots i_{m}=1 \\
\delta_{i_{j} i_{2} \ldots i_{m}=0}}}^{n} \mathcal{E}_{i_{j} i_{2} \ldots i_{m}}\left(\frac{x_{\text {min }}}{x_{\max }}\right)^{m-1}\right) \\
& \leq \prod_{j=1}^{m}\left(\rho(\mathcal{B})-b_{i_{j} i_{j} \ldots i_{j}}-(\tau(\mathcal{B}))^{m-1} \sum_{\substack{i_{2}, \ldots i_{m}=1 \\
\delta_{i_{j} i_{2} \ldots i_{m}=0}}}^{n} \mathcal{E}_{i_{j} i_{2} \ldots i_{m}}\right) \\
& =\prod_{j=1}^{m}\left(\rho(\mathcal{B})-b_{i_{j} i_{j} \ldots i_{j}}-(\tau(\mathcal{B}))^{m-1} r_{i_{j}}(\mathcal{E})\right),
\end{aligned}
$$

where the last inequality follows from (8).

Similarly, using Theorem 4 and the method of proof employed in Theorem 10, we can obtain improvement of (2) as follows:

Corollary 2 Let $\mathcal{A}=\left(a_{i_{1} i_{2} \ldots i_{m}}\right)$ and $\mathcal{B}=\left(b_{i_{1} i_{2} \ldots i_{m}}\right)$ be complex tensors of order $m$ dimension $n$ with $|\mathcal{A}| \leq \mathcal{B}$. Then, every eigenvalue of $\mathcal{A}$ lies in the region:

$$
\begin{aligned}
& \bigcup_{\substack{i, j=1 \\
i \neq j}}^{n}\left\{z:\left|z-a_{i \ldots i}\right|^{m-1}\left|z-a_{j \ldots j}\right| \leq\left(\rho(\mathcal{B})-b_{i \ldots i}-(\tau(\mathcal{B}))^{m-1} r_{i}(\mathcal{E})\right)^{m-1}\right. \\
& \left.\left(\rho(\mathcal{B})-b_{j \ldots j}-(\tau(\mathcal{B}))^{m-1} r_{j}(\mathcal{E})\right)\right\}:=\mathfrak{B}_{2}(\mathcal{A}) .
\end{aligned}
$$

In the following, we consider the relationships between Ky Fan theorem and its improvements.

Theorem 11 Let $\mathcal{A}$ be tensor of order $m$ dimension $n$, and then:

$$
\mathcal{Z}_{1}(\mathcal{A}) \subseteq \mathfrak{B}_{1}(\mathcal{A}) \subseteq \mathcal{K}(\mathcal{A})
$$

Proof First, consider the right-hand inclusion of (12). Fix any $i, j(1 \leq i, j \leq n ; i \neq j)$ and let $z$ be any point of $\mathfrak{B}_{1}^{(i, j)}(\mathcal{A})$, where:

$$
\mathfrak{B}_{1}^{(i, j)}(\mathcal{A}):=\left|z-a_{i i \ldots i}\right|^{m-1}\left|z-a_{j j \ldots j}\right| \leq\left(\rho(\mathcal{B})-b_{i i \ldots i}\right)^{m-1}\left(\rho(\mathcal{B})-b_{j j \ldots j}\right) .
$$

By Corollary 1, we have:

$$
\left|z-a_{i \ldots i}\right|^{m-1}\left|z-a_{j \ldots j}\right| \leq\left(\rho(\mathcal{B})-b_{i \ldots i}\right)^{m-1}\left(\rho(\mathcal{B})-b_{j \ldots j}\right), \quad i, j \in\langle n\rangle, i \neq j .
$$

If $\left(\rho(\mathcal{B})-b_{i i \ldots i}\right)^{m-1}\left(\rho(\mathcal{B})-b_{j j \ldots j}\right)=0$, then:

$$
z=a_{i i \ldots i} \quad \text { or } \quad z=a_{j j \ldots j} .
$$


Since $a_{i i \ldots i} \in \mathcal{K}^{(i)}(\mathcal{A})$ and $a_{j j \ldots j} \in \mathcal{K}^{(j)}(\mathcal{A})$, then:

$$
z \in \mathcal{K}^{(i)}(\mathcal{A}) \cup \mathcal{K}^{(j)}(\mathcal{A}) .
$$

If $\left(\rho(\mathcal{B})-b_{i i \ldots i}\right)^{m-1}\left(\rho(\mathcal{B})-b_{j j \ldots j}\right)>0$, then by (13), we have:

$$
\left(\frac{\left|z-a_{i i \ldots . i}\right|}{\rho(\mathcal{B})-b_{i i \ldots i}}\right)^{m-1}\left(\frac{\left|z-a_{j j \ldots j}\right|}{\rho(\mathcal{B})-b_{j j \ldots j}}\right) \leq 1 .
$$

As the factors on the left-hand of (14) cannot all exceed unity, then at least one of these factors is unity, at most, i.e., $z \in \mathcal{K}^{(i)}(\mathcal{A}) \cup \mathcal{K}^{(j)}(\mathcal{A})$. Hence, in either case, it follows that $z \in \mathcal{K}^{(i)}(\mathcal{A}) \cup \mathcal{K}^{(j)}(\mathcal{A})$. Therefore:

$$
\mathfrak{B}_{1}(\mathcal{A}) \subseteq \bigcup_{i=1}^{n} \mathcal{K}^{(i)}(\mathcal{A})=\mathcal{K}(\mathcal{A}) .
$$

Next, we prove that the left-hand inclusion of (12) is valid. Using the first part of Theorem 7 , we have:

$$
\left(\rho(\mathcal{B})-b_{i i \ldots i}\right)>0, \quad \forall i \in\langle n\rangle .
$$

Let $z$ be any point of $\mathcal{Z}_{1}(\mathcal{A})$, that is:

$$
\prod_{j=1}^{m}\left|z-a_{i_{j} \ldots i_{j}}\right| \leq \prod_{j=1}^{m}\left(\rho(\mathcal{B})-b_{i_{j} \ldots i_{j}}\right) .
$$

On taking the power of $m$ for the inequality (15), we have:

$$
\prod_{j=1}^{m}\left|z-a_{i_{j} \ldots i_{j}}\right|^{m} \leq \prod_{j=1}^{m}\left(\rho(\mathcal{B})-b_{i_{j} \ldots i_{j}}\right)^{m} .
$$

Since $\left(\rho(\mathcal{B})-b_{i_{j} \ldots i_{j}}\right)$ are all positive for $j=1,2, \ldots, m$, we may equivalently express the inequality (16) in the following form:

$$
\begin{aligned}
& \left(\frac{\left|z-a_{i_{1} \ldots i_{1}}\right|^{m-1}\left|z-a_{i_{2} \ldots i_{2}}\right|}{\left(\rho(\mathcal{B})-b_{i_{1} \ldots i_{1}}\right)^{m-1}\left(\rho(\mathcal{B})-b_{i_{2} \ldots i_{2}}\right)}\right) \cdot\left(\frac{\left|z-a_{i_{2} \ldots i_{2}}\right|^{m-1}\left|z-a_{i_{3} \ldots i_{3}}\right|}{\left(\rho(\mathcal{B})-b_{i_{2} \ldots i_{2}}\right)^{m-1}\left(\rho(\mathcal{B})-b_{i_{3} \ldots i_{3}}\right)}\right) \\
& \quad \ldots\left(\frac{\left|z-a_{i_{1} . i_{m}}\right|^{m-1}\left|z-a_{i_{1} \ldots i_{1}}\right|}{\left(\rho(\mathcal{B})-b_{i_{m} \ldots i_{m}}\right)^{m-1}\left(\rho(\mathcal{B})-b_{i_{1} \ldots i_{1}}\right)}\right) \leq 1 .
\end{aligned}
$$

As the factors on the left of (17) cannot all exceed unity, then at least one of these factors is unity, at most. That is:

$$
\left(\frac{\left|z-a_{i_{1} \ldots i_{1}}\right|^{m-1}\left|z-a_{i_{2} \ldots i_{2}}\right|}{\left(\rho(\mathcal{B})-b_{i_{1} \ldots i_{1}}\right)^{m-1}\left(\rho(\mathcal{B})-b_{i_{2} \ldots i_{2}}\right)}\right) \leq 1
$$

or

$$
\left(\frac{\left|z-a_{i_{2} \ldots i_{2}}\right|^{m-1}\left|z-a_{i_{3} \ldots i_{3}}\right|}{\left(\rho(\mathcal{B})-b_{i_{2} \ldots i_{2}}\right)^{m-1}\left(\rho(\mathcal{B})-b_{i_{3} \ldots i_{3}}\right)}\right) \leq 1
$$

or 
or

$$
\left(\frac{\left|z-a_{i_{m} \ldots i_{m}}\right|^{m-1}\left|z-a_{i_{1} \ldots i_{1}}\right|}{\left(\rho(\mathcal{B})-b_{i_{m} \ldots i_{m}}\right)^{m-1}\left(\rho(\mathcal{B})-b_{i_{1} \ldots i_{1}}\right)}\right) \leq 1 .
$$

Hence, there exists an $\alpha(1<\alpha<m)$, such that:

$$
\left|z-a_{i_{\alpha} \ldots i_{\alpha}}\right|^{m-1}\left|z-a_{i_{\alpha+1} \ldots i_{\alpha+1}}\right| \leq\left(\rho(\mathcal{B})-b_{i_{\alpha} \ldots i_{\alpha}}\right)\left(\rho(\mathcal{B})-b_{i_{\alpha+1} \ldots i_{\alpha+1}}\right) ;
$$

that is, $z \in \mathfrak{B}_{1}^{(\alpha, \alpha+1)}(\mathcal{A})$. Therefore, by definition of $\mathfrak{B}_{1}(\mathcal{A})$, we have $z \in \mathfrak{B}_{1}(\mathcal{A})$. Thus, $\mathcal{Z}_{1}(\mathcal{A}) \subseteq \mathfrak{B}_{1}(\mathcal{A})$. Therefore, the proof is complete.

Corollary 3 Let $\mathcal{A}$ be tensor of order $m$ dimension $n$, and then:

$$
\mathcal{Z}_{2}(\mathcal{A}) \subseteq \mathcal{Z}_{1}(\mathcal{A}) \subseteq \mathfrak{B}_{1}(\mathcal{A}) \subseteq \mathcal{K}(\mathcal{A})
$$

Remark 4 Using the method of proof employed in Theorem 11, we conclude:

$$
\mathcal{Z}_{2}(\mathcal{A}) \subseteq \mathfrak{B}_{2}(\mathcal{A}) .
$$

The following examples show the improvements of the bounds obtained in this section.

Example 1 Let $\mathcal{A}=\left(a_{i j k}\right)$ be a tensor of order 3 dimension 2 with entries defined as follows:

$$
a_{111}=a_{122}=a_{211}=1, a_{222}=-1 \text { and other } a_{i j k}=0 .
$$

Let $\mathcal{B}=\left(a_{i j k}\right)$ be a tensor of order 3 dimension 2 , where $\mathcal{B}=|\mathcal{A}|$. By computation (Chang et al. 2008), we have $\sigma(\mathcal{A})=\{\sqrt{2},-\sqrt{2}\}$, and $\rho(\mathcal{B})=2$.

By Ky Fan theorem (Theorem 6), we obtain:

$$
\sigma(\mathcal{A}) \subseteq \mathcal{K}(\mathcal{A}):=\{z:|z-1| \leq(2-1)\} \cup\{z:|z+1| \leq(2-1)\} .
$$

From Corollary 1, we obtain:

$$
\sigma(\mathcal{A}) \subseteq \mathfrak{B}_{1}(\mathcal{A}):=\left\{z:|z-1|^{2}|z+1| \leq(2-1)^{3}\right\} \cup\left\{z:|z+1|^{2}|z-1| \leq(2-1)^{3}\right\} .
$$

Also from Theorem 7, we obtain:

$$
\sigma(\mathcal{A}) \subseteq \mathcal{Z}_{1}(\mathcal{A}):=\left\{z:|z-1||z+1| \leq(2-1)^{2}\right\} .
$$

The blue line in Fig. 1 shows the boundary of $\mathcal{K}(\mathcal{A})$ (Ky Fan theorem), the yellow line shows the boundary of $\mathfrak{B}_{1}(\mathcal{A})$ (Corollary 1 ), and the red line shows the boundary of $\mathcal{Z}_{1}(\mathcal{A})$ (Theorem 7). This example shows that $\mathcal{Z}_{1}(\mathcal{A}) \subseteq \mathfrak{B}_{1}(\mathcal{A})$.

Remark 5 In Example $1, \mathcal{E}=0(\mathcal{B}=|\mathcal{A}|)$, and therefore, the eigenvalue inclusion sets $\mathcal{Z}_{1}(\mathcal{A})$ and $\mathcal{Z}_{2}(\mathcal{A})$ are equal. In the following example, we consider $\mathcal{B} \supsetneqq|\mathcal{A}|$.

Example 2 Let $\mathcal{A}=\left(a_{i j k}\right)$ be an order 3 dimension 2 tensor with entries defined as follows:

$$
a_{111}=a_{122}=a_{211}=1, \quad a_{222}=-1 \text { and other } a_{i j k}=0 .
$$

Let $\mathcal{B}=\left(b_{i j k}\right)$ be an order 3 dimension 2 tensor, where $b_{i j k}=2$ for nay $1 \leq i, j, k \leq 2$. By Yang and Yang (2010, Lemma 5.1), we have $\rho(\mathcal{B})=8$.

From Corollary 2, we obtain:

$$
\mathfrak{B}_{2}(\mathcal{A}):=\left\{z:|z-1|^{2}|z+1| \leq(8-2-5 \times 1)^{3}(8-2-5 \times 1)\right\}
$$




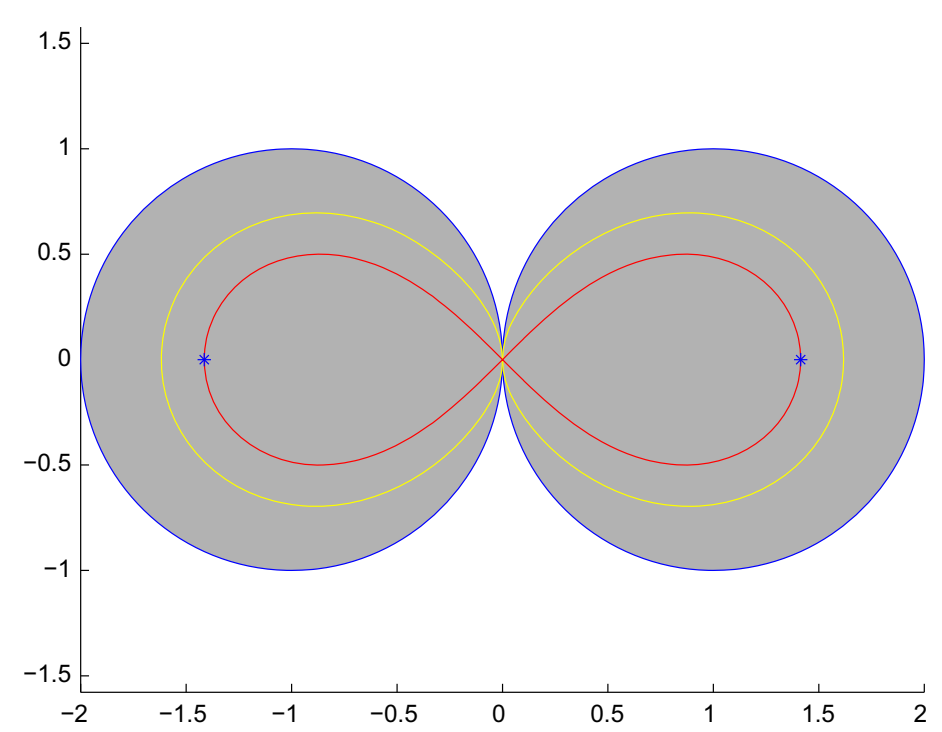

Fig. 1 Comparisons of Ky Fan theorem, Corollary 2, and Theorem 7. The "*” symbols show the location of the eigenvalues of $\mathcal{A}$

$$
\cup\left\{z:|z+1|^{2}|z-1| \leq(8-2-5 \times 1)^{3}(8-2-5 \times 1)\right\} .
$$

Also from Theorem 10, we obtain:

$$
\sigma(\mathcal{A}) \subseteq \mathcal{Z}_{2}(\mathcal{A}):=\left\{z:|z-1||z+1| \leq(8-2-5 \times 1)^{2}\right\} .
$$

However, from Theorem 4.6 of Wang et al. (2020) (Theorem 9), we obtain:

$$
\sigma(\mathcal{A}) \subseteq\left\{z:|z-1||z+1| \leq(8-2)^{2}-(5 \times 1)^{2}\right\} .
$$

It is clear that $\mathcal{Z}_{2}(\mathcal{A})$ is strictly better that the bounds $\mathfrak{B}_{2}(\mathcal{A})$ and (19).

\subsection{New improvements of Ky Fan theorem}

In this section, we obtain another improvement of Ky Fan theorem. First, we prove the following lemma that will be needed.

Lemma 2 Let $\mathcal{A}=\left(a_{i_{1} i_{2} \ldots i_{m}}\right)$ be a tensor of order $m$ dimension $n$. For any eigenvalue $\lambda$ of $\mathcal{A}$, there exits a nonzero $x \in \mathbb{C}^{n}$, such that:

$$
\left(\lambda-a_{s \ldots s}\right)\left(\lambda-\sum_{\substack{i_{2}, \ldots, i_{m}=1 \\ \delta_{s i_{2} \ldots i_{m}}=0}}^{n} a_{t i_{2} \ldots i_{m}} \frac{x_{i_{2}} \ldots x_{i_{m}}}{x_{t}^{m-1}}\right)=a_{t s \ldots s} \sum_{\substack{i_{2}, \ldots, i_{m}=1 \\ \delta_{s i_{2} \ldots i_{m}=0}=0}}^{n} a_{s i_{2} \ldots i_{m}} \frac{x_{i_{2}} \ldots x_{i_{m}}}{x_{t}^{m-1}},
$$

for all $s \in\langle n\rangle$ and $t \in T$, where $T=\left\{t \in\langle n\rangle: t \neq s, x_{t} \neq 0\right\}$.

Proof Let $\lambda$ be eigenvalue of $\mathcal{A}$ with the eigenvector $x$, that is:

$$
\mathcal{A} x^{m-1}=\lambda x^{[m-1]} \text {. }
$$


For each $s \in\langle n\rangle$ and $t \in T$, we have:

$$
\begin{aligned}
\left(\lambda-a_{s s \ldots s}\right) x_{s}^{m-1} & =\sum_{\substack{i_{2}, \ldots, i_{m}=1 \\
\delta_{s i_{2} \ldots i_{m}}=0}}^{n} a_{s i_{2} \ldots i_{m}} x_{i_{2}} \ldots x_{i_{m}}, \\
\left(\lambda-a_{t t \ldots t}\right) x_{t}^{m-1} & =\sum_{\substack{\delta_{t i_{2} \ldots i_{m}}=0 \\
\delta_{s i_{2} \ldots i_{m}}=0}}^{n} a_{t i_{2} \ldots i_{m}} x_{i_{2}} \ldots x_{i_{m}}+a_{t s \ldots s} x_{s}^{m-1} .
\end{aligned}
$$

If $\left(\lambda-a_{s s . . . s}\right) \neq 0$ by (22), one has:

$$
\begin{aligned}
& \left(\lambda-a_{s s \ldots s}\right)\left(\lambda-a_{t t \ldots t}\right) x_{t}^{m-1}-\sum_{\substack{\delta_{t i_{2} \ldots i_{m}}=0 \\
\delta_{s i_{2} \ldots i_{m}}=0}}\left(\lambda-a_{s s \ldots s}\right) a_{t i_{2} \ldots i_{m}} x_{i_{2}} \ldots x_{i_{m}} \\
& =\left(\lambda-a_{s s \ldots s}\right) a_{t s \ldots s} x_{s}^{m-1} .
\end{aligned}
$$

Also if $a_{t s . . s} \neq 0$ by (21), one has:

$$
\left(\lambda-a_{s s \ldots s}\right) a_{t s . . s} x_{s}^{m-1}=a_{t s \ldots s}\left(\sum_{\begin{array}{l}
\delta_{s i_{2} \ldots i_{m}=0}=0 \\
\delta_{t i_{2} \ldots i_{m}}=0
\end{array}} a_{s i_{2} \ldots i_{m}} x_{i_{2} \ldots} x_{i_{m}}+a_{s t \ldots t} x_{t}^{m-1}\right) .
$$

By (23) and (24), we have:

$$
\begin{aligned}
& \left(\lambda-a_{s s \ldots s}\right)\left(\left(\lambda-a_{t t \ldots t}\right)-\sum_{\substack{\delta_{s i_{2} \ldots i_{m}}=0 \\
\delta_{t i_{2} \ldots i_{m}}=0}} a_{t i_{2} \ldots i_{m}} \frac{x_{i_{2} \ldots x_{i_{m}}}}{x_{t}^{m-1}}\right) \\
& =a_{t s \ldots s}\left(\sum_{\substack{\delta_{s i_{2} \ldots m_{m}=0}=0 \\
\delta_{t i_{2} \ldots i_{m}}=0}} a_{s i_{2} \ldots i_{m}} \frac{x_{i_{2} \ldots x_{i_{m}}}}{x_{t}^{m-1}}+a_{s t \ldots t}\right),
\end{aligned}
$$

where is equal to:

$$
\left(\lambda-a_{s s \ldots s}\right)\left(\lambda-\sum_{\substack{i_{2}, \ldots, i_{m}=1 \\ \delta_{s i_{2} \ldots i_{m}=0}}}^{n} a_{t i_{2} \ldots i_{m}} \frac{x_{i_{2} \ldots x_{i_{m}}}}{x_{t}^{m-1}}\right)=a_{t s \ldots s} \sum_{\substack{i_{2}, \ldots, i_{m}=1 \\ \delta_{s i_{2} \ldots i_{m}=0}}}^{n} a_{s i_{2} \ldots i_{m}} \frac{x_{i_{2} \ldots x_{i_{m}}}}{x_{t}^{m-1}} .
$$

If $\left(\lambda-a_{s s \ldots s}\right), a_{t s \ldots s}=0$, then the relation (20) is also valid for this case in fact. Thus, the proof is complete.

Theorem 12 Let $\mathcal{A}$ and $\mathcal{B}$ be tensor of order $m$ dimension $n$ with $|\mathcal{A}| \leq \mathcal{B}$, and then:

$$
\sigma(\mathcal{A}) \subseteq \bigcap_{s \in N}\left(\bigcup_{\substack{t \in N \\ s \neq t}} \mathcal{O}_{s, t}\right):=\mathcal{K}_{s, t}(\mathcal{A})
$$


where

$$
\begin{aligned}
\mathcal{O}_{s, t} & =\left\{z \in \mathbb{C}:\left|z-\frac{a_{s \ldots s}+a_{t \ldots t}}{2}\right|\right. \\
& \left.\leq \frac{1}{2}\left|\rho_{t}(\mathcal{B})+\sqrt{\left(\left|a_{s \ldots . s}-a_{t \ldots t}\right|+\rho_{t}(\mathcal{B})\right)^{2}+4 b_{t s \ldots s} \rho_{s}(\mathcal{B})}\right|\right\}
\end{aligned}
$$

$\rho_{t}(\mathcal{B})=\rho(\mathcal{B})-b_{t t \ldots t}-b_{t s \ldots s}\left(\frac{u_{s}}{u_{t}}\right)^{m-1}-r_{t}^{s}(\mathcal{E})\left(\frac{u_{\min }}{u_{t}}\right)^{m-1}$,

$\rho_{s}(\mathcal{B})=\left(\rho(\mathcal{B})-b_{s s . . . s}\right)\left(\frac{u_{s}}{u_{t}}\right)^{m-1}-r_{s}(\mathcal{E})\left(\frac{u_{\min }}{u_{t}}\right)^{m-1}$, and $u$ is a Perron vector of $\mathcal{B}$.

Proof Similarly by proof of Lemma 2, we define:

$$
z_{i}=\frac{\left|x_{i}\right|}{u_{i}}, \quad i \in\langle n\rangle, \quad i \neq s
$$

Let

$$
T^{\prime}:=\max _{\substack{j \in\langle n\rangle \\ j \neq s}}\left\{j: z_{j}=\frac{\left|x_{j}\right|}{u_{j}}\right\} ;
$$

it is evident that $T^{\prime} \subseteq T$. For all $s \in\langle n\rangle$ and $t \in T^{\prime}$, we set:

$$
\alpha_{s}=\sum_{\substack{i_{2}, \ldots, i_{m}=1 \\ \delta_{s i_{2} \ldots i_{m}}=0}}^{n} a_{s i_{2} \ldots i_{m}} \frac{x_{i_{2} \ldots x_{i_{m}}}}{x_{t}^{m-1}}
$$

and

$$
\alpha_{t}=\sum_{\substack{i_{2}, \ldots, i_{m}=1 \\ \delta_{s i_{2} \ldots i_{m}}=0}}^{n} a_{t i_{2} \ldots i_{m}} \frac{x_{i_{2} \ldots i_{m}}}{x_{t}^{m-1}}=\sum_{\substack{i_{2}, \ldots, i_{m}=1 \\ \delta_{s i_{2} \ldots i_{m}}=0 \\ \delta_{t i_{2} \ldots i_{m}}=0}}^{n} a_{t i_{2} \ldots i_{m}} \frac{x_{i_{2} \ldots} \ldots x_{i_{m}}}{x_{t}^{m-1}}+a_{t t \ldots t} .
$$

Since $u$ is a Perron vector of the positive tensor $\mathcal{B}$, so:

$$
\mathcal{B} u^{m-1}=\rho(\mathcal{B}) u^{[m-1]} .
$$

Let $\mathcal{E}=\mathcal{B}-|\mathcal{A}|$, and then, the triangle inequality permits us to conclude that:

$$
\begin{aligned}
\left|\alpha_{s}\right| & \leq \sum_{\substack{i_{2}, \ldots, i_{m}=1 \\
\delta_{s i_{2} \ldots i_{m}}=0}}^{n}\left|a_{s i_{2} \ldots i_{m}}\right|\left|\frac{x_{i_{2}} \ldots x_{i_{m}}}{x_{t}^{m-1}}\right| \\
& =\sum_{\substack{i_{2}, \ldots, i_{m}=1 \\
\delta_{s i_{2} \ldots i_{m}}=0}}^{n}\left(b_{s i_{2} \ldots i_{m}}-e_{s i_{2} \ldots i_{m}}\right)\left|\frac{\left(z_{i_{2}} u_{i_{2}}\right) \ldots\left(z_{i_{m}} u_{i_{m}}\right)}{\left(z_{t} u_{t}\right)^{m-1}}\right| \\
& \leq \sum_{\substack{i_{2}, \ldots, i_{m}=1 \\
\delta_{s i_{2} \ldots m_{m}}=0}}^{n} b_{s i_{2} \ldots i_{m}} \frac{u_{i_{2} \ldots} \ldots u_{i_{m}}}{u_{t}^{m-1}}-\sum_{\substack{i_{2}, \ldots, i_{m}=1 \\
\delta_{s i_{2} \ldots i_{m}}=0}}^{n} e_{s i_{2} \ldots i_{m}} \frac{u_{i_{2} \ldots u_{i_{m}}}}{u_{t}^{m-1}} \\
& \leq \frac{1}{u_{t}^{m-1}} \sum_{i_{2}, \ldots, i_{m}=1}^{n} b_{s i_{2} \ldots i_{m}} u_{i_{2} \ldots u_{i_{m}}}-b_{s s \ldots s} u_{s}^{m-1}-\left(\frac{u_{m i n}}{u_{t}}\right)^{m-1} r_{s}(\mathcal{E})
\end{aligned}
$$




$$
=\left(\frac{u_{s}}{u_{t}}\right)^{m-1}\left(\rho(\mathcal{B})-b_{s s \ldots s}\right)-\left(\frac{u_{m i n}}{u_{t}}\right)^{m-1} r_{s}(\mathcal{E}) \equiv \rho_{s}(\mathcal{B}),
$$

and

$$
\begin{aligned}
& \left|\alpha_{t}-a_{t t \ldots t}\right| \leq \sum_{\substack{i_{2}, \ldots, i_{m}=1 \\
\delta_{s i_{2} \ldots i_{m}}=0 \\
\delta_{t i_{2} \ldots i_{m}}=0}}^{n}\left|a_{t i_{2} \ldots i_{m}}\right|\left|\frac{x_{i_{2} \ldots i_{i_{m}}}}{x_{t}^{m-1}}\right| \\
& =\sum_{\substack{i_{2}, \ldots, i_{m}=1 \\
\delta_{s i_{2} \ldots i_{m}=0}=0 \\
\delta_{t i_{2} \ldots i_{m}}=0}}^{n}\left(b_{t i_{2} \ldots i_{m}}-e_{t i_{2} \ldots i_{m}}\right)\left|\frac{\left(z_{i_{2}} u_{i_{2}}\right) \ldots\left(z_{i_{m}} u_{i_{m}}\right)}{\left(z_{t} u_{t}\right)^{m-1}}\right| \\
& \leq \sum_{\substack{i_{2}, \ldots, i_{m}=1 \\
\delta_{s i_{2} \ldots i_{m}}=0 \\
\delta_{t i_{2} \ldots i_{m}=0}=0}}^{n} b_{t i_{2} \ldots i_{m}} \frac{u_{i_{2} \ldots} \ldots u_{i_{m}}}{u_{t}^{m-1}}-\sum_{\substack{i_{2}, \ldots, i_{m}=1 \\
\delta_{s i_{2} \ldots i_{m}=0}=0 \\
\delta_{t i_{2} \ldots i_{m}}=0}}^{n} e_{t i_{2} \ldots i_{m}} \frac{u_{i_{2}} \ldots u_{i_{m}}}{u_{t}^{m-1}} \\
& \leq \sum_{\substack{i_{2}, \ldots, i_{m}=1 \\
\delta_{s i_{2} \ldots i_{m}}=0}}^{n} b_{t i_{2} \ldots i_{m}} \frac{u_{i_{2} \ldots u_{i_{m}}}}{u_{t}^{m-1}}-b_{t t \ldots t}-\left(\frac{u_{m i n}}{u_{t}}\right)^{m-1} r_{t}^{s}(\mathcal{E}) \\
& =\rho(\mathcal{B})-b_{t t \ldots t}-b_{t s \ldots s}\left(\frac{u_{s}}{u_{t}}\right)^{m-1}-\left(\frac{u_{m i n}}{u_{t}}\right)^{m-1} r_{t}^{s}(\mathcal{E}) \equiv \rho_{t}(\mathcal{B}),
\end{aligned}
$$

where $r_{t}^{s}(\mathcal{E})$ is defined in (1). By Lemma 2, we have:

$$
\left(\lambda-a_{s s \ldots s}\right)\left(\lambda-\alpha_{t}\right)=a_{t s \ldots s} \alpha_{s} .
$$

Therefore:

$$
\lambda=\frac{\left(\alpha_{t}+a_{s s \ldots s}\right) \pm \sqrt{\left(\alpha_{t}+a_{s s \ldots s}\right)^{2}-4\left(a_{s s \ldots s} \alpha_{t}-a_{t s \ldots s} \alpha_{s}\right)}}{2} .
$$

Using the inequalities (27) and (28), we obtain:

$$
\begin{aligned}
\left|\lambda-\frac{\left(a_{s s \ldots s}+a_{t t \ldots t}\right)}{2}\right| & =\left|\frac{\left(\alpha_{t}-a_{t t \ldots t}\right) \pm \sqrt{\left(a_{s s \ldots s}-\alpha_{t}\right)^{2}-4 a_{t s \ldots s} \alpha_{s}}}{2}\right| \\
& \leq \frac{1}{2}\left|\rho_{t}(\mathcal{B})+\sqrt{\left(\left|a_{s \ldots . s}-a_{t \ldots t}\right|+\rho_{t}(\mathcal{B})\right)^{2}+4 b_{t s \ldots s} \rho_{s}(\mathcal{B})}\right| .
\end{aligned}
$$

Since $s$ is arbitrary, we have:

$$
\sigma(\mathcal{A}) \subseteq \bigcap_{s \in\langle n\rangle}\left(\bigcup_{\substack{t \in\langle n\rangle \\ t \neq s}} \mathcal{O}_{s, t}\right)
$$

The proof of the theorem is complete. 
Theorem 13 Let $\mathcal{A}, \mathcal{B}$ be tensor of order $m$ dimension $n$ with $|\mathcal{A}| \leq \mathcal{B}$, and then:

$$
\sigma(\mathcal{A}) \subseteq \bigcap_{s \in N}\left(\bigcup_{\substack{t \in N \\ s \neq t}} \mathcal{M}_{s, t}\right):=\mathcal{K}_{2}(\mathcal{A})
$$

where

$$
\mathcal{M}_{s, t}=\left\{z \in \mathbb{C}:\left|z-a_{s s \ldots s}\right|\left|z-a_{t t \ldots t}\right| \leq\left|z-a_{s s \ldots s}\right| \rho_{t}(\mathcal{B})+b_{t s \ldots s} \rho_{s}(\mathcal{B})\right\},
$$

and $\rho_{t}(\mathcal{B}), \rho_{s}(\mathcal{B})$ are the same as those in Theorem 12 .

Proof First, for all $s \in\langle n\rangle$ and $t \in T^{\prime}$, according to the definition of $T^{\prime}$, we have $T^{\prime} \subseteq T$. We write Eq. (20) in the form:

$$
\begin{aligned}
& \lambda^{2}-\lambda\left(\sum_{\substack{i_{2} \ldots i_{m} \in\langle n\rangle \\
\delta_{s i_{2} \ldots i_{m}=0}=0 \\
\delta_{t i_{2} \ldots i_{m}=0}}} a_{t i_{2} \ldots i_{m}} \frac{x_{i_{2} \ldots x_{i_{m}}}}{x_{t}^{m-1}}+a_{t t \ldots t}\right)-a_{s \ldots s} \lambda \\
& +a_{s \ldots s}\left(\sum_{\substack{i_{2} \ldots i_{m} \in\langle n\rangle \\
\delta_{s i_{2} \ldots i_{m}=0}=0 \\
\delta_{t i_{2} \ldots i_{m}=0}}} a_{t i_{2} \ldots i_{m}} \frac{x_{i_{2} \ldots x_{i_{m}}}}{x_{t}^{m-1}}+a_{t t \ldots t}\right. \\
& =a_{t s \ldots s} \sum_{\substack{i_{2} \ldots i_{m} \in\langle n\rangle \\
\delta_{s i_{2} \ldots i_{m}=0}=0}} a_{s i_{2} \ldots i_{m}} \frac{x_{i_{2} \ldots x_{i_{m}}}}{x_{t}^{m-1}} .
\end{aligned}
$$

Therefore, we have:

$$
\begin{aligned}
& \left(\lambda-a_{s s \ldots s}\right)\left(\lambda-a_{t t \ldots t}\right) \\
& =\left(\lambda-a_{s s \ldots s}\right) \sum_{\substack{i_{2} \ldots i_{m} \in\langle n\rangle \\
\delta_{s i_{2} \ldots i_{m}=0}=0 \\
\delta_{t i_{2} \ldots i_{m}=0}=0}} a_{t i_{2} \ldots i_{m}} \frac{x_{i_{2} \ldots x_{i_{m}}}}{x_{t}^{m-1}}+a_{t s \ldots s} \sum_{\substack{i_{2} \ldots i_{m} \in\langle n\rangle \\
\delta_{s i_{2} \ldots i_{m}}=0}} a_{s i_{2} \ldots i_{m}} \frac{x_{i_{2}} \ldots x_{i_{m}}}{x_{t}^{m-1}} .
\end{aligned}
$$

Using the inequalities (27) and (28), we have:

$$
\left|\lambda-a_{s s \ldots s}\right|\left|\lambda-a_{t t \ldots t}\right| \leq\left|\lambda-a_{s s \ldots s}\right| \rho_{t}(\mathcal{B})+\rho_{s}(\mathcal{B}) b_{t s \ldots s},
$$

i.e., $\lambda \in \mathcal{M}_{s, t}$. Since $s$ is arbitrary, we have:

$$
\sigma(\mathcal{A}) \subseteq \bigcap_{s \in N}\left(\bigcup_{\substack{t \in N \\ s \neq t}} \mathcal{M}_{s, t}\right):=\mathcal{K}_{2}(\mathcal{A})
$$

By estimating the ratio of the smallest component and the largest component of the Perron vector of $\mathcal{B}$ (Lemma 1), the following result is obtained. 
Theorem 14 Let $\mathcal{A}, \mathcal{B}$ be tensor of orderm dimension n with $|\mathcal{A}| \leq \mathcal{B}$. Then, everyeigenvalue of $\mathcal{A}$ lies in the region:

$$
\bigcap_{i \in\langle n\rangle}\left(\bigcup_{\substack{j \in\langle n\rangle \\ i \neq j}} \tilde{\mathcal{M}}_{i, j}\right):=\tilde{\mathcal{K}}_{2}(\mathcal{A}),
$$

where

$$
\tilde{\mathcal{M}}_{i, j}:=\left\{z \in \mathbb{C}:\left|z-a_{i i \ldots i}\right|\left|z-a_{j j \ldots j}\right| \leq\left|z-a_{i i \ldots i}\right| \tilde{\rho}_{j}(\mathcal{B})+b_{j i \ldots i} \tilde{\rho}_{i}(\mathcal{B})\right\},
$$

$\tilde{\rho}_{j}(\mathcal{B})=\rho(\mathcal{B})-b_{j j \ldots j}-(\tau(\mathcal{B}))^{m-1}\left(b_{j i \ldots i}+r_{j}^{i}(\mathcal{E})\right)$,

$\tilde{\rho}_{i}(\mathcal{B})=\left(\rho(\mathcal{B})-b_{i i \ldots i}\right)\left(\frac{1}{\tau(\mathcal{B})}\right)^{m-1}-(\tau(\mathcal{B}))^{m-1} r_{i}(\mathcal{E})$, and $\tau(\mathcal{B})$ is given in $(8)$.

Wang et al. (2020) in Example 4.11 show that their bounds are better than the bound (10). By the same example, we show that our bounds are better than others given in the literature.

Example 3 (Wang et al. 2020, Example 4.11) Consider 3 order 2 dimensional tensors $\mathcal{A}=$ $\left(a_{i j k}\right)$ and $\mathcal{B}=\left(b_{i j k}\right)$ defined by:

$$
\begin{aligned}
& a_{i j k}=\left\{\begin{array}{l}
a_{111}=0 ; \quad a_{112}=0 ; \quad a_{121}=0 ; \quad a_{122}=-1 ; \\
a_{211}=-4 ; \quad a_{212}=0 ; \quad a_{221}=0 ; \quad a_{222}=0 ;
\end{array}\right. \\
& b_{i j k}=\left\{\begin{array}{llll}
b_{111}=1 ; & b_{112}=\frac{1}{4} ; & b_{121}=\frac{1}{4} ; & b_{122}=2 ; \\
b_{211}=4 ; & b_{212}=\frac{1}{2} ; & b_{221}=\frac{1}{2} ; & b_{222}=\frac{3}{2}
\end{array}\right.
\end{aligned}
$$

Obviously, $\mathcal{B} \geq|\mathcal{A}|$ and $\mathcal{A}$ is weakly irreducible. By computations (Liu and Chen 2019), we can calculate:

$$
\{\rho(\mathcal{B}), x\}=\{4.8095,(0.6930,0.8738)\}, \quad r_{1}(\mathcal{E})=\frac{3}{2}, \quad r_{2}(\mathcal{E})=1,
$$

where $\mathcal{E}=\mathcal{B}-|\mathcal{A}|$. From Theorem 3.3 of $\mathrm{Li}$ and $\mathrm{Ng}(2015)$, we may compute $\kappa(\mathcal{B})=\frac{5}{18}$ and:

$$
|\lambda| \leq 4.8095-1-\left(\frac{5}{18}\right)^{2} \times \frac{3}{2}=3.6938 .
$$

From Theorem 4.6 of Wang et al. (2020), we may compute $P=\max \left\{\left(\frac{5}{18}\right)^{2}, \frac{1}{4}, 0.1603\right\}=$ $\frac{1}{4}$ and:

$$
|\lambda| \leq \sqrt{(4.8095-1) \times\left(4.8095-\frac{3}{2}\right)-\left(\frac{1}{4}\right)^{2} \times \frac{3}{2} \times 1}=3.5375 .
$$

By Theorem 4.7 of Wang et al. (2020) (Theorem 7), we get:

$$
|\lambda| \leq \sqrt{(4.8095-1) \times\left(4.8095-\frac{3}{2}\right)}=3.5507 .
$$

From Theorem 4.9 of Wang et al. (2020), we obtain:

$$
|\lambda| \leq 4.8095-1-\frac{1}{4} \times \frac{3}{2}=3.4345 .
$$


From Lemma 1, we may compute $\tau(\mathcal{B})=\max \left\{\left(\frac{5}{18}\right)^{2},\left(\frac{1}{2}\right)^{2}\right\}=\frac{1}{4}$, and therefore, by Theorem 10, we have:

$$
|\lambda| \leq \sqrt{\left[4.8095-1-\left(\frac{1}{4} \times \frac{3}{2}\right)\right] \times\left[4.8095-\frac{3}{2}-\left(\frac{1}{4} \times 1\right)\right]}=3.2416 .
$$

From Corollary 2, we obtain:

$$
|\lambda| \leq\left(\left[4.8095-1-\left(\frac{1}{4} \times \frac{3}{2}\right)\right]^{2} \times\left[4.8095-\frac{3}{2}-\left(\frac{1}{4} \times 1\right)\right]\right)^{\frac{1}{3}}=3.3047 .
$$

From Theorem 12 by $s=2, t=1$, we may compute:

$$
\begin{aligned}
\rho_{1}(\mathcal{B}) & =(4.8095-1)-2 \times\left(\frac{0.8738}{0.6930}\right)^{2}-\frac{1}{2} \times\left(\frac{0.6930}{0.6930}\right)^{2}=0.1298 \\
\rho_{2}(\mathcal{B}) & =\left(4.8095-\frac{3}{2}\right) \times\left(\frac{0.8738}{0.6930}\right)^{2}-1 \times\left(\frac{0.6930}{0.6930}\right)^{2}=4.2616, \text { and } \\
|\lambda| & \leq \frac{1}{2}\left[0.1298+\sqrt{(0.1298)^{2}+4 \times 2 \times 4.2616}\right]=2.9851 .
\end{aligned}
$$

Also from Theorem 13 by $s=2, t=1$, we obtain:

$$
|\lambda|^{2} \leq|\lambda| \times 0.1298+2 \times 4.2616
$$

which this eigenvalue inclusion set is equal to $|\lambda| \leq 2.9851$. This example shows that if we use the ratio of the smallest and largest entries of the Perron vector $(\tau(\mathcal{B}))$, then the bound of Theorem 10 is tighter than other bounds. However, if there exist the perron vector of $\mathcal{B}$, then the bounds of Theorems 12 and 13 are tighter.

\section{Conclusion}

In this paper, we proposed some new improvements of Ky Fan theorem for tensors. We obtain new lower bound for the ratio of the smallest and largest entries of the Perron vector using a technique due to Wang et al. (2020) and Li and $\mathrm{Ng}$ (2015). Based on these new lower bounds, we obtain Theorems 10 and 12, which improve the bounds in $\mathrm{Li}$ and $\mathrm{Ng}$ (2015) and Wang et al. (2020). Each of these new bounds is always better than the existing one. Several numerical examples are given to show that our bounds are better than others given in the literature.

Open Access This article is licensed under a Creative Commons Attribution 4.0 International License, which permits use, sharing, adaptation, distribution and reproduction in any medium or format, as long as you give appropriate credit to the original author(s) and the source, provide a link to the Creative Commons licence, and indicate if changes were made. The images or other third party material in this article are included in the article's Creative Commons licence, unless indicated otherwise in a credit line to the material. If material is not included in the article's Creative Commons licence and your intended use is not permitted by statutory regulation or exceeds the permitted use, you will need to obtain permission directly from the copyright holder. To view a copy of this licence, visit http://creativecommons.org/licenses/by/4.0/. 


\section{References}

Brauer A (1947) Limits for the characteristic roots of a matrix II. Duke Math J 14(1):21-26

Bu C, Jin X, Li H, Deng C (2017) Brauer-type eigenvalue inclusion sets and the spectral radius of tensors. Linear Algebra Appl 512:234-248

Chang KC, Pearson K, Zhang T (2008) Perron-Frobenius theorem for nonnegative tensors. Commun Math Sci 6(2):507-520

Fan K (1958) Note on circular disks containing the eigenvalues of a matrix. Duke Math J 25(3):441-445

Horn RA, Johnson CR (2012) Matrix analysis. Cambridge University Press, Cambridge

Li HB, Huang TZ (2005) An improvement of Ky Fan theorem for matrix eigenvalues. Comput Math Appl 49(5-6):789-803

Li C, Li Y (2016) An eigenvalue localization set for tensors with applications to determine the positive (semi-) definiteness of tensors. Linear Multilinear Algebra 64(4):587-601

Li C, Li Y (2016) Relationships between Brauer-type eigenvalue inclusion sets and a Brualdi-type eigenvalue inclusion set for tensors. Linear Algebra Appl 496:71-80

Li W, Ng MK (2015) Some bounds for the spectral radius of nonnegative tensors. Numer Math 130(2):315-335

Li C, Li Y, Kong X (2014) New eigenvalue inclusion sets for tensors. Numer Linear Algebra Appl 21(1):39-50

Li S, Chen Z, Li C, Zhao J (2020) Eigenvalue bounds of third-order tensors via the minimax eigenvalue of symmetric matrices. Comput Appl Math 39:217

Liu Q, Chen Z (2019) An algorithm for computing the spectral radius of nonnegative tensors. Comput Appl Math 38(2):90

Qi L (2005) Eigenvalues of a real supersymmetric tensor. J Symb Comput 40(6):1302-1324

Qi L, Luo Z (2017) Tensor analysis: spectral theory and special tensors. SIAM, Philadelphia

Varga RS (2004) Gershgorin and his circles. Springer Series in Computational Mathematics, New York

Wang G, Wang Y, Wang Y (2020) Some Ostrowski-type bound estimations of spectral radius for weakly irreducible nonnegative tensors. Linear Multilinear Algebra 68(9):1817-1834

Wei Y, Ding W (2017) Theory and computation of tensors: multi-dimensional arrays. Academic Press, London

Xu Y, Zheng B, Zhao R (2019) Some results on Brauer-type and Brualdi-type eigenvalue inclusion sets for tensors. Comput Appl Math 38:74

Yang Y, Yang Q (2010) Further results for Perron-Frobenius theorem for nonnegative tensors. SIAM J Matrix Anal Appl 31(5):2517-2530

Publisher's Note Springer Nature remains neutral with regard to jurisdictional claims in published maps and institutional affiliations. 